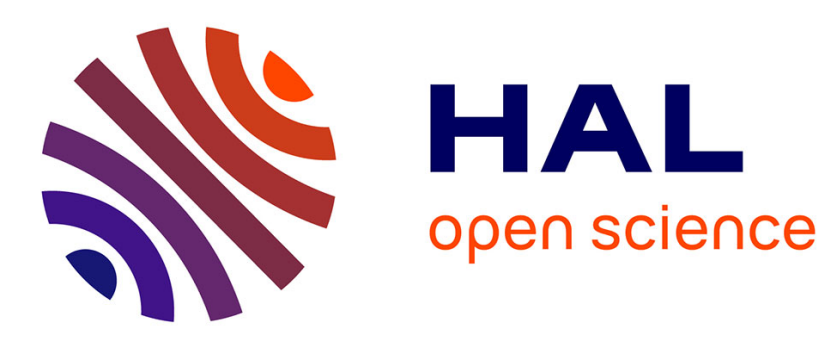

\title{
Polymorphism versus devitrification mechanism: Low-wavenumber Raman investigations in sulindac
}

Mansour Latreche, Jean-François Willart, Laurent Paccou, Yannick Guinet, Alain Hedoux

\section{- To cite this version:}

Mansour Latreche, Jean-François Willart, Laurent Paccou, Yannick Guinet, Alain Hedoux. Polymorphism versus devitrification mechanism: Low-wavenumber Raman investigations in sulindac. International Journal of Pharmaceutics, 2019, International Journal of Pharmaceutics, 567, pp.118476. 10.1016/j.ijpharm.2019.118476 . hal-02363464

\section{HAL Id: hal-02363464 https://hal.science/hal-02363464}

Submitted on 16 Nov 2020

HAL is a multi-disciplinary open access archive for the deposit and dissemination of scientific research documents, whether they are published or not. The documents may come from teaching and research institutions in France or abroad, or from public or private research centers.
L'archive ouverte pluridisciplinaire HAL, est destinée au dépôt et à la diffusion de documents scientifiques de niveau recherche, publiés ou non, émanant des établissements d'enseignement et de recherche français ou étrangers, des laboratoires publics ou privés. 
Polymorphism versus devitrification mechanism: low-wavenumber Raman investigations in sulindac

Mansour Latreche, Jean-François Willart, Laurent Paccou, Yannick Guinet and Alain Hédoux

Univ. Lille, CNRS, INRA, ENSCL, UMR 8207 - UMET - Unité Matériaux et

Transformations, F-59000 Lille, France

\begin{abstract}
The polymorphism of Sulindac was investigated by Raman investigations, mainly in the lowwavenumber region in order to analyze the influence of the amorphization method on recrystallization and crystalline form stability. By devitrification of the quenched liquid, it was found that the undercooled liquid crystallizes into Form I, and a polymorphic transformation by cooling Form I toward Form IV, was clearly revealed. The lowwavenumber spectra of polymorphic forms are direct fingerprints of crystals, indicating a degree of disorder of Form IV intermediate between those of the ordered Form II (commercial form) and the relatively disordered Form I. This study has shown the enantiotropic relationship between Forms I and IV and that both the temperature of crystallization and the physical stability of Form I prepared is dependent on the technique used for preparing amorphous sulindac.
\end{abstract}

Keywords: low-wavenumber Raman spectroscopy - active pharmaceutical ingredient polymorphism - amorphous - devitrification mechanism

Corresponding author: Alain Hédoux, alain.hedoux@univ-lille.fr 


\section{INTRODUCTION}

Organic molecular compounds are materials characterized by a significant disparity between weak intermolecular interactions and much stronger intramolecular interactions. This property is responsible for a rich polymorphism in this class of materials often characterized by different molecular associations via $\mathrm{H}$-bonding, and these materials can be easily vitrified by cooling the liquid state or by milling crystalline states below $T_{g}$ (Willart and Descamps, 2008). Slight temperature or pressure changes can easily induce phase transformation between polymorphs, and devitrification mechanisms are highly dependent on the route of amorphization(Guinet et al., 2016). In the pharmaceutical area, the knowledge of polymorphs and polymorphic transformations is crucial for the drug formulation. It was shown that lowwavenumber Raman spectroscopy (LWRS) is a powerful technique for analyzing devitrification mechanisms and polymporphic transformations (Malfait et al., 2019). The temperature dependence of the quasielastic intensity, detected at very low wavenumbers, provides information on the mechanisms of phase transformations and the degree of disorder of transient metastable states (Guinet et al., 2016; Malfait et al., 2019) detected just above $T_{g}$ along the ordering process toward the ultimately ordered phase. Additionally, the analysis of collective motions in amorphous (vibrational density of states, $G(\omega)$ ) and crystalline (lattice modes or phonon peaks) states allows the monitoring of the molecular organization along the ordering process (Malfait et al., 2019). The spectrum of the lattice modes is the crystalline signature of polymorphs closely related to the spatial group of the crystalline state (Hédoux et al., 2013; Hédoux et al., 2009). The development of a new generation of filters with holographic gratings (Larkin et al., 2014) enables measurements of low-wavenumber spectra from a relatively compact and easy to use system. In this context, LWRS becomes extensively used in the pharmaceutical area (Hisada et al., 2015; Koide et al., 2016; Shibata et al., 2015). In this paper we are concerned with sulindac, a nonsteroidal anti-inflammatory drug which can exist in three polymorphic forms: Forms I (CCDC number DOHREX01 Deposition Number 637252), II (CCDC code DOHREX Deposition Number 1143612) and Form IV (CCDC code DOHREX03 Deposition Number 625435) which have respectively the space groups $\mathrm{P} 2{ }_{1} / \mathrm{c}$ (Llinàs et al., 2007) and $\mathrm{Pbca}$ (Koo et al., 1985) and P2 $1 / \mathrm{c}$ (Grzesiak and Matzger, 2007). Using LWRS, the polymorphism of sulindac was explored from devitrification of amorphous states obtained either by quenching the liquid or by milling commercial Form II of sulindac, in order to analyze the influence of the amorphization method on recrystallization and subsequent polymorphic transformations.

\section{Material and methods}

\section{Sample}

Crystalline sulindac was provided by SIGMA ${ }^{\circledR}$ life science (purity $\leq 98 \%$ ) and used as received without any further purification. The as-received material is in the crystalline Form II. Sulindac $\left(\mathrm{C}_{20} \mathrm{H}_{17} \mathrm{~F} \mathrm{O}_{3} \mathrm{~S}\right)$ is a nonsteroidal anti-inflammatory agent with a rich polymorphism.

\section{Instruments}


Ball milling was performed with a high energy planetary mill (Pulverisette 7 - Fritsch) using $\mathrm{ZrO}_{2}$ milling jars of $43 \mathrm{~cm}^{3}$ with seven balls $(\varnothing=15 \mathrm{~mm})$ of the same material. $1 \mathrm{~g}$ of sulindac powder was placed in the planetary mill corresponding to a ball:sample weight ratio of 75:1. The rotation speed of the solar disk was set to $400 \mathrm{rpm}$ which corresponds to an average acceleration of the milling balls of $5 \mathrm{~g}$ ( $\mathrm{g}=9.81 \mathrm{~ms}^{-2}$ is the acceleration of gravity). We took care to alternate milling periods (typically $10 \mathrm{~min}$ ) with pause periods (typically $5 \mathrm{~min}$ ) in order to limit the mechanical heating of the sample.

Differential Scanning Calorimetry (DSC) experiments were performed with a Q20 micro calorimeter of TA Instruments. During all the measurements the calorimeter head was flushed with highly pure nitrogen gas. Temperature and enthalpy readings were calibrated using pure indium at the same scan rates used in the experiments. All DSC scans (heating) reported in the figures were performed at the rate of $0,5^{\circ} \mathrm{C} / \mathrm{min}$. The samples have been placed in open pans (pans with no lid) in order to allow any water absorbed during the milling process to evaporate upon heating. As recommended by the manufacturer small sample size (typically 4 mg) were used to achieve good resolution and good thermal conductivity.

Raman investigations were performed in the low-wavenumber region using a triple monochromator XY Dilor spectrometer. It is a high dispersive system making possible to analyze Raman signal down to $5 \mathrm{~cm}^{-1}$ using the $647.3 \mathrm{~nm}$ radiation of an argon-krypton Coherent laser. Powder samples were loaded in capillary pyrex cells hermetically sealed. The temperature of sample was regulated using an Oxford nitrogen flux device that keeps temperature fluctuations within $0.1^{\circ} \mathrm{C}$. The acquisition time was systematically 2 minutes. The cooling and heating runs of amorphous or crystalline sulindac were analyzed by collecting low-wavenumber spectra during a heating ramp at $\dot{T}= \pm 0.5{ }^{\circ} \mathrm{C} / \mathrm{min}$. Raman investigations were also performed in the molecular fingerprint region using an InVia Renishaw Raman microscope. Spectra were collected in the $400-1800 \mathrm{~cm}^{-1}$ spectral range using the $785 \mathrm{~nm}$ line from a Renishaw diode. Spectra were collected during heating and cooling runs with the same acquisition time with a THMS 600 Linkam temperature device.

\section{Raman analysis in the low-wavenumber region}

The analysis of low-wavenumber Raman spectrum (LWRS) of disordered molecular materials, considering molecules as rigid bodies, requires a two steps processing of Raman data. The first step consists to transform the Raman intensity into reduced intensity $\mathrm{I}_{\mathrm{r}}(\omega)$ according to (Galeener and Sen, 1978; Shuker and Gammon, 1970):

$$
I_{r}(\omega)=\frac{I_{R a m a n}(\omega, T)}{[n(\omega, T)+1] \omega}
$$

where $n(\omega, T)$ is the Bose-Einstein factor. This transformation provides the low-wavenumber Raman band shape free of temperature fluctuation. The second step is the fitting procedure described in figure 1, used for determining the two overlapped contributions corresponding to two kinds of molecular motions. The first class corresponds to semi internal/external motions, i.e. large amplitude rotations of a group of atoms within the molecule or the whole molecule giving a contribution to the very low-wavenumber Raman scattering, the so called quasi- 
elastic scattering (QES). This type of motions is closely related to order-disorder phase transformations, and their analysis provides information on the mechanism of phase transformations. The QES contribution to the LWRS is generally described by a Lorentzian function centered at zero. It is the dominant contribution in the reduced intensity spectrum, strongly temperature dependent as expected for anharmonic motions (Hédoux, 2016).

The second class corresponds to collective motions providing information on the structural organization of molecules from the short-range order in amorphous states to the long-range order in the crystals. The analysis of this contribution requires removing the QES contribution before the transformation of the reduced intensity into Raman susceptibility according to:

$$
\chi^{\prime \prime}(\omega)=\omega \cdot I_{r}(\omega)
$$

The Raman susceptibility is recognized to be a representation very close to the vibrational density of states (VDOS, $G(\omega)$ ) providing the vibrational signature to the structural organization in highly disordered states including amorphous states (Hedoux et al., 2001). The lowest wavenumber vibrational component plotted in dashed line in Figure 1 corresponds to the Boson peak, i.e. the universal low-wavenumber vibrational signature of amorphous states. For crystalline states, $\chi^{\prime \prime}(\omega)$ spectrum is composed of lattice modes characterizing the crystalline fingerprint of polymorphs.

The vibrational contribution to the LWRS has weak linear temperature dependence, closely related to the thermal expansion of the sample. Consequently $\mathrm{I}_{\mathrm{r}}(\omega)$ spectra were normalized by the integrated intensity in the $70-150 \mathrm{~cm}^{-1}$, corresponding to the spectral containing only collective vibrational bands. The quasielastic intensity ( $\mathrm{I}_{\mathrm{QES}}$ ) was obtained by integrating the reduced intensity in the $10-30 \mathrm{~cm}^{-1}$ range, dominated by the QES.

The $500-1600 \mathrm{~cm}^{-1}$ fingerprint region was analyzed, but only investigations around 1600 $\mathrm{cm}^{-1}$ composed of most intense Raman bands were presented for more clarity, since it was shown that polymorphic forms of sulindac can be identified in the $1550-1700 \mathrm{~cm}^{-1}$ spectral range (Grzesiak and Matzger, 2007). The analysis of the whole fingerprint region performed after quenching liquid sulindac below $T_{g}$, and after milling has shown similar spectra for both amorphous states reached via different routes. This indicates the same molecular conformation in both amorphous states, without signature of chemical degradation. Additionally, the spectra of the fingerprint region in various forms analyzed in the present study are identical to those presented in a previous paper (Grzesiak and Matzger, 2007), including the spectrum of Form IV, obtained from transformation of recrystallized Form I in the present study and directly prepared by polymer-induced heteronucleation (Grzesiak and Matzger, 2007). It is another indication that no change in chemical bonds which have a Raman signature in the $500-1600 \mathrm{~cm}^{-1}$ region was detected after heating Form II above $\mathrm{T}_{\mathrm{m}}$, after quenching liquid sulindac below $T_{g}$, heating the glassy state up to crystallization in Form I, and after cooling Form I toward Form IV.

\section{RESULTS AND DISCUSSION}

\section{Devitrification of quenched liquid into Form I.}


Phase II (as received sulindac) was firstly heated above $\mathrm{T}_{\mathrm{m}}$ and rapidly cooled down to room temperature by shifting the nose of the Oxford device. The low-wavenumber spectrum collected after quenching liquid sulindac at room temperature is presented in Figure 1. In a second step, low-wavenumber spectra were collected during heating from 20 up to $170{ }^{\circ} \mathrm{C}$. The temperature dependence of the quasielastic intensity was determined as described in the previous section and plotted in Figure 2. $\mathrm{I}_{\mathrm{QES}}(\mathrm{T})$ is compared to the DSC trace obtained after the same thermal history and upon the same heating $\operatorname{ramp}\left(\dot{T}=0.5^{\circ} \mathrm{C} / \mathrm{min}\right)$. Figure 2 shows that the glass transition is detected from Raman data via a change in the slope of the quasielastic intensity (Guinet et al., 2016; Malfait et al., 2019), roughly corresponding to the $\mathrm{C}_{\mathrm{p}}$ jump observed from DSC data. The change in the slope of $\mathrm{I}_{\mathrm{QES}}(\mathrm{T})$ indicating a change in the dynamics of sulindac at $T_{g}$, was previously observed for other molecular materials (Guinet et al., 2016; Malfait et al., 2019), and then can be considered as the Raman signature of the glass transition. Upon further heating a drastic decrease of $\mathrm{I}_{\mathrm{QES}}$ is observed above $140{ }^{\circ} \mathrm{C}$, corresponding to the crystallization of the undercooled liquid into Form I of sulindac. Surprisingly, no crystallization was detected from the DSC trace. This could be induced by a softer quenching procedure used within the DSC apparatus. In Raman investigations the rapid quenching of the liquid can generate cracks in the glassy state thereby promoting nucleation, not in the less rapid cooling process within the furnace of the DSC apparatus. This feature could also indicate a possible influence of the shape of the sample container on the devitrification mechanism.

\section{Evidence for $1^{\text {st }}$ order polymorphic transition.}

After obtaining Form I, low-wavenumber Raman spectra were collected during a cooling ramp at $\dot{T}=-0.5^{\circ} \mathrm{C} / \mathrm{min}$. Spectra were plotted in Figure $3 \mathrm{a}$ in the reduced intensity in the $65--50{ }^{\circ} \mathrm{C}$ temperature range where a significant change in the low-wavenumber band shape was observed. This change can be mainly described as a discontinuity in the position of the dominant Raman band in the reduced intensity representation. The reversible transformation in the low-wavenumber band shape is observed upon heating in Figure $3 b$. This discontinuous change in the low-wavenumber band shape reveals a first-order phase transformation between two polymorphs of sulindac. A fitting procedure with mixed Gaussian-Lorentzian shapes was used for analyzing the temperature dependence of the position of the dominant lowwavenumber band. The temperature dependence of the position of this band, plotted in Figure 4 , reveals that the $1^{\text {st }}$ order phase transition is characterized by a large hysteresis induced by the coexistence of Forms I and the low-temperature form, in agreement with the temperature dependence of $\mathrm{I}_{\mathrm{r}}(\omega)$ spectra plotted in Figures $3 \mathrm{a}$ and $3 \mathrm{~b}$. It was shown that sulindac crystallizes in solution in form IV under specific conditions (Grzesiak and Matzger, 2007). This crystalline form was characterized by X-ray diffraction and by Raman spectroscopy in the fingerprint region. To identify the form of sulindac detected upon cooling Form I around $\mathrm{T}=-20{ }^{\circ} \mathrm{C}$, Raman spectra were collected in the fingerprint region using the same conditions than for the low-wavenumber analysis. Spectra are plotted in Figure 5a, only around $1600 \mathrm{~cm}^{-}$ ${ }^{1}$ for better clarity, since this spectral region allows the identification of polymorphic forms of sulindac. A discontinuity is clearly observed around $-20{ }^{\circ} \mathrm{C}$ for Raman bands around $1630 \mathrm{~cm}$ ${ }^{1}$ producing a spectrum similar to that of Form IV in this region (Grzesiak and Matzger, 
2007). Consequently, the polymorphic form observed by cooling Form I down to $-20{ }^{\circ} \mathrm{C}$ can be assigned to Form IV revealed by Grzesiak et al. The spectra of various polymorphic forms of sulindac plotted in Figure $5 \mathrm{~b}$ in the $1600 \mathrm{~cm}^{-1}$ region at $20^{\circ} \mathrm{C}$, are in good agreement with those collected by Grzesiak et al.(Grzesiak and Matzger, 2007).

\section{Structural description of polymorphic forms in sulindac}

It is well known that the low-wavenumber region corresponds to the crystalline fingerprint of polymorphic forms and gives direct information about the degree of disorder in crystalline states via the number of lattice modes and the low-wavenumber band shape(Hédoux, 2016). For obtaining information about the structural organization in the crystalline and amorphous states, the reduced intensity was converted into Raman susceptibility.

In a first step, Raman susceptibilities of Forms I and II are compared in relation with that of the amorphous state at room temperature in Figure 6a. It is clearly observed that the spectrum of the glassy state roughly corresponds to the envelope of the crystalline Forms I and II. Comparing spectra of Forms I and II shows that Form I is more disordered than the commercial Form II, since it is clearly observed that the number of Raman bands in Form I is smaller and Raman bands in Form I are broader than in Form II.

In a second step, Raman susceptibilities of the three crystalline forms are compared in Figure 6b. It can be observed that the spectrum of Forms IV resembles the spectrum of Form II, except that the lowest wavenumber band in Form II does not exist in Form IV. Consequently the degree of disorder of Form IV can be considered as intermediate between Forms I and II.

The shape of Raman bands in the fingerprint region is closely dependent on the close molecular neighboring. Consequently it is clearly observed in Figure $5 \mathrm{~b}$ that devitrification induced a crystalline form (Form I) characterized by a molecular packing close to the shortrange order of the amorphous state. Form IV appears as an intermediate between the relatively disordered Form I and the ultimately ordered Form II, in agreement with the low-wavenumber analysis.

\section{Influence of the devitrification mechanism on FI to FIV transformation}

The polymorphic Form I to Form IV transformation was analyzed from Form I obtained by recrystallization of amorphous sulindac prepared by milling at room temperature for 600 minutes. The Raman spectrum of the amorphous state obtained by milling is rigorously similar to that obtained by quenching the liquid state. Raman spectra were collected during a heating ramp for analyzing devitrification of the amorphous state obtained by milling. Using processing of Raman spectra similar to that used for analyzing devitrification of the quenched liquid, allows the comparison of the two devitrification mechanisms via the plot of $\mathrm{I}_{\mathrm{QES}}(\mathrm{T})$ in Figure 7. This figure firstly reveals different values of the quasielastic intensity and different temperature behaviors in the glassy state, with a less marked change in the slope for the milled sample observed at lower temperature $\left(\sim 50^{\circ} \mathrm{C}\right)$. The low contribution of the fast dynamics in the milled amorphous powder can be explained from the consideration that fast $\beta$ motions exhibit a low degree of cooperativity(Fragiadakis and Roland, 2018; Ngai and 
Paluch, 2004). In this context, the grain size could limit intermolecular cooperativity and thereby the contribution to this fast dynamics in the quasielastic intensity detected in Raman spectroscopy. In the undercooled liquid $\mathrm{I}_{\mathrm{QES}}(\mathrm{T})$ curves become superimposed. Secondly, recrystallization in Form I was detected at $\mathrm{T}_{\mathrm{c}} \sim 70{ }^{\circ} \mathrm{C}$, i.e. about $70{ }^{\circ} \mathrm{C}$ below recrystallization from the quenched liquid. This indicates a weaker contribution of rapid $\beta$-motions in milled amorphous powder at Tc compared with quenched liquid.

Form I obtained by devitrification of the amorphous milled powder was analyzed upon cooling. Spectra during cooling ramp down to $-100{ }^{\circ} \mathrm{C}$ at $\dot{T}=-0.5^{\circ} \mathrm{C} / \mathrm{min}$. $\mathrm{I}_{\mathrm{r}}(\omega)$ spectra calculated from spectra collected during the cooling run are plotted in Figure 8 between 100 and $-100{ }^{\circ} \mathrm{C}$. This figure clearly reveals the absence of phase transition, since no change is detected in the Raman spectrum of Form I, except the decrease of the quasielastic intensity. This absence of Form I to Form IV transition from Form I obtained by devitrification of the amorphous milled powder can be explained from the observation of slight differences in $\chi$ ” $(\omega)$ spectra of Form I obtained by devitrification of the two amorphous states, i.e. quenched liquid and amorphous powder, plotted in Figure 9. The spectrum of Form I recrystallized from the amorphous powder has a band shape broader than that of Form I recrystallized from quenched liquid, reflecting a lower degree of crystallization. This indicates that recrystallization from the amorphous powder obtained by milling Form II results in a not completely achieved long-range order. The poor degree of crystallinity of Form I obtained by devitrification of milled amorphous powder could explain that the Form I to Form IV transformation is inhibited. The low degree of crystallinity of Form I resulting from devitrification of the amorphous powder can be interpreted from investigations of devitrification of indomethacin (IMC) amorphized by milling (Hédoux et al., 2009) by micro calorimetry. An isothermal crystallization of the amorphous powder was detected about $20{ }^{\circ} \mathrm{C}$ below $T_{g}$ determined as uncomplete. Upon heating, the crystallization of the residual amorphous powder was observed via a sharp exothermic peak, followed by a broad exothermic feature associated to a mechanism of coalescence. This shows in some cases, the facility of the amorphous milled powder to recrystallize, and also the difficulty to completely recrystallize. Both features could be related to a crystallization mechanism from incipient nuclei and limited to the scale of the grain size.

\section{CONCLUSION}

Low-wavenumber investigations have revealed a polymorphic transformation between Forms I and IV in sulindac in the $-20{ }^{\circ} \mathrm{C}-40{ }^{\circ} \mathrm{C}$ temperature range. Form IV appears as an intermediate crystalline state between Forms II and I in the ordering process from the amorphous state toward the stable Form II. It was found that Form IV can be only obtained by cooling Form I resulting from devitrification of quenched liquid. It was shown that the devitrification mechanism of the milled amorphous state is different from that of quenched liquid, as observed in other pharmaceuticals (Guinet et al., 2016), probably because of the lower temperature of crystallization. In these conditions, the long-range order of Form I is not complete that could induce the suppression of the transformation of Form I toward Form IV. The present study also reveals that Form IV can be prepared otherwise than by polymer- 
induced nucleation (Grzesiak and Matzger, 2007). Additionally, the enantiotropic relationship between Form I and Form IV was clearly shown, contrarily to monotropic relationship revealed by the study of Grzesiak et al. when Form IV is directly prepared by polymerinduced heteronucleation (Grzesiak and Matzger, 2007).

\section{ACKNOWLEDGEMENTS}

This project has received funding from the Interreg 2 Seas programme 2014-2020 co-funded by the European Regional Development Fund under subsidy contract 2S01-059_IMODE 


\section{REFERENCES}

Fragiadakis, D., Roland, C., 2018. Cooperativity of short-time dynamics revisited. J. Non-Cryst. Sol. $500,18-21$.

Galeener, F.L., Sen, P.N., 1978. Theory for the first-order vibrational spectra of disordered solids. Physical Review B 17, 1928-1933.

Grzesiak, A., Matzger, A.J., 2007. New form discovery for the analgesics flurbiprofen and sulindac facilitated by polymer-induced heteronucleation. J. Pharm. Sci. 96, 2978-2986.

Guinet, Y., Paccou, L., Danede, F., Willart, J.F., Derollez, P., Hédoux, A., 2016. Comparison of amorphous states prepared by melt-quenching and cryomilling polymorphs of carbamazépine. Int. J. Pharm. 509, 305-313.

Hédoux, A., 2016. Recent developments in the Raman and infrared investigations of amorphous pharmaceuticals and protein formulations: a review. Adv. Drug Deliv. Rev. 100, 133-146.

Hedoux, A., Derollez, P., Guinet, Y., Dianoux, A.J., Descamps, M., 2001. Low-frequency vibrational excitations in the amorphous and crystalline states of triphenyl phosphite: A neutron and Raman scattering investigation Physical Review B 63, 144202-144210.

Hédoux, A., Guinet, Y., Paccou, L., Derollez, P., Danede, F., 2013. Vibrational and structural properties of amorphous n-butanol: A complementary Raman spectroscopy and X-ray diffraction study. J. Chem. Phys. 138, 214506-214501 - 2145068.

Hédoux, A., Paccou, L., Guinet, Y., Willart, J.-F., Descamps, M., 2009. Using the low-frequency Raman spectroscopy to analyze the crystallization of amorphous indomethacin. European Journal of Pharmaceutical Sciences 38, 156-164.

Hisada, H., Inoue, M., Koide, T., Carriere, J., Heyler, R., Fukami, T., 2015. Direct high-resolution imaging of crystalline components in pharmaceutical dosage forms using low-frequency Raman spectroscopy. Org. Process Res. Dev. 19, 1796-1798.

Koide, T., Fukami, T., Hisada, H., Inoue, M., Carriere, J., Heyler, R., Katori, N., Okuda, H., Goda, Y., 2016. Identification of pseudopolymorphism of magnesium stearate by using low-frequency Raman spectroscopy. Org. Process Res. Dev. 20, 1906-1910.

Koo, C., Kim, S., Shin, W., 1985. Crystal structure of antiinflammatory sulindac. Bul. Korean Chem. Soc. 6, 222-224.

Larkin, P.J., Dabros, M., Sarsfield, B., Chan, E., Carriere, J., Smith, B.C., 2014. Polymorph characterization of active pharmaceutical ingredients (APIs) using low-frequency Raman spectroscopy. Appl. Spectr. 68, 758-776.

Llinàs, A., Box, K., Burley, J., Glen, R., Goodman, J., 2007. A new method for the reproducible generation of polymorphs: two forms of sulindac with very different solubilities. J. Appl. Cryst. 40, 379-381.

Malfait, B., Paccou, L., derollez, P., Guinet, Y., Hedoux, A., 2019. Capabilities of low-wavenumber Raman spectroscopy for analyzing the mechanism of devitrification of molecular glasses. J. Raman Spectr. DOI: $10.1002 /$ jrs.5606.

Ngai, K., Paluch, M., 2004. Classification of secondary relaxation in glass-formers based on dynamic properties. J. Chem. Phys. 120, 857-873.

Shibata, T., Mori, T., Kojima, S., 2015. Low-frequency vibrational properties of crystalline and glassy indomethacin probed by terahertz time-domain spectroscopy and low-frequency Raman scattering. Spectrochimica Acta Part A 150, 207-211.

Shuker, R., Gammon, R., 1970. Raman-scattering selection-rule breaking and the density of states in amorphous materials. Physical Review Letters 25, 222-225.

Willart, J.-F., Descamps, M., 2008. Solid State Amorphization of Pharmaceuticals. Molecular Pharmaceutics 5, 905-920. 
Figures

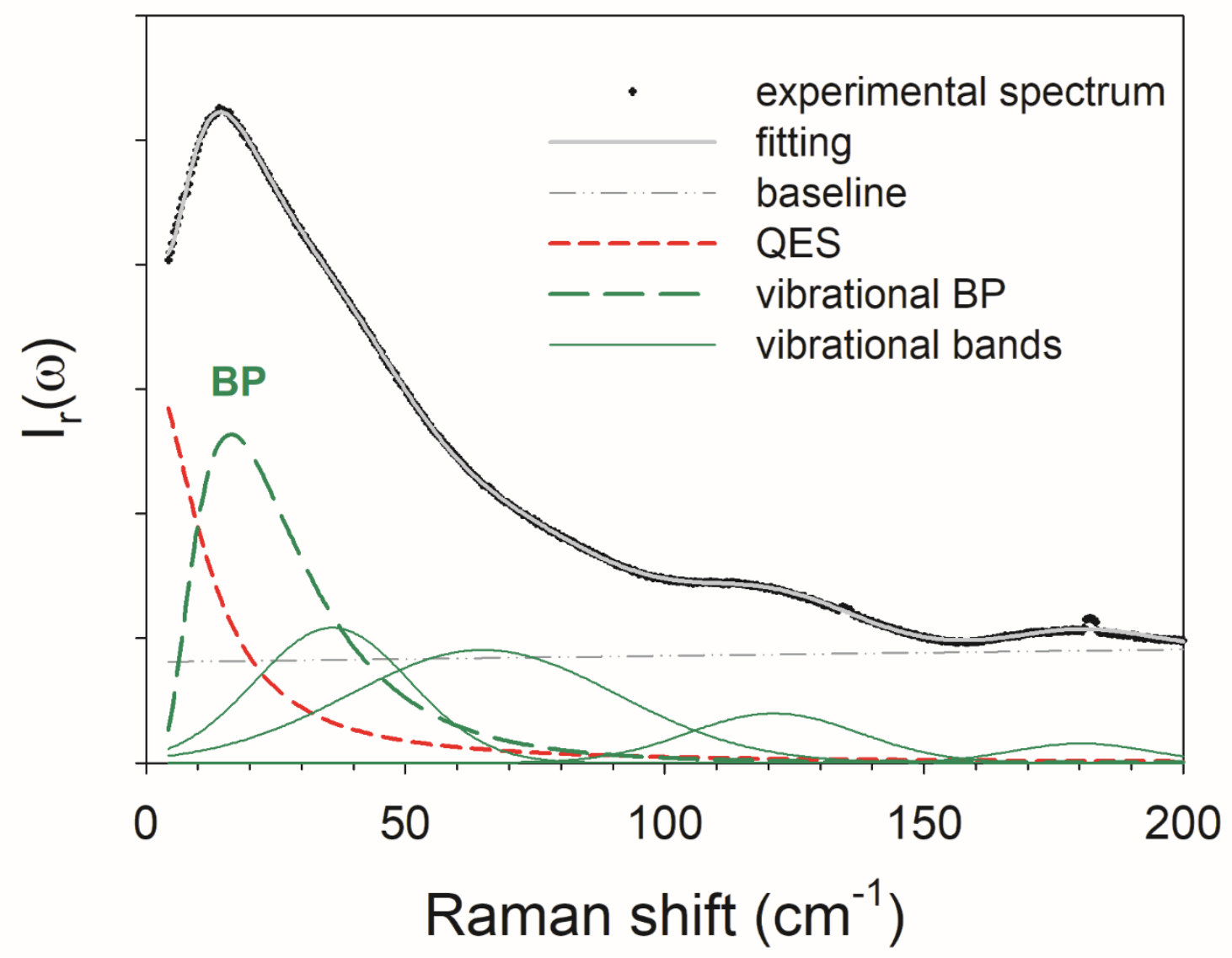

Figure 1: description of the fitting procedure used to analyze separately the quasielastic and the vibrational contributions 


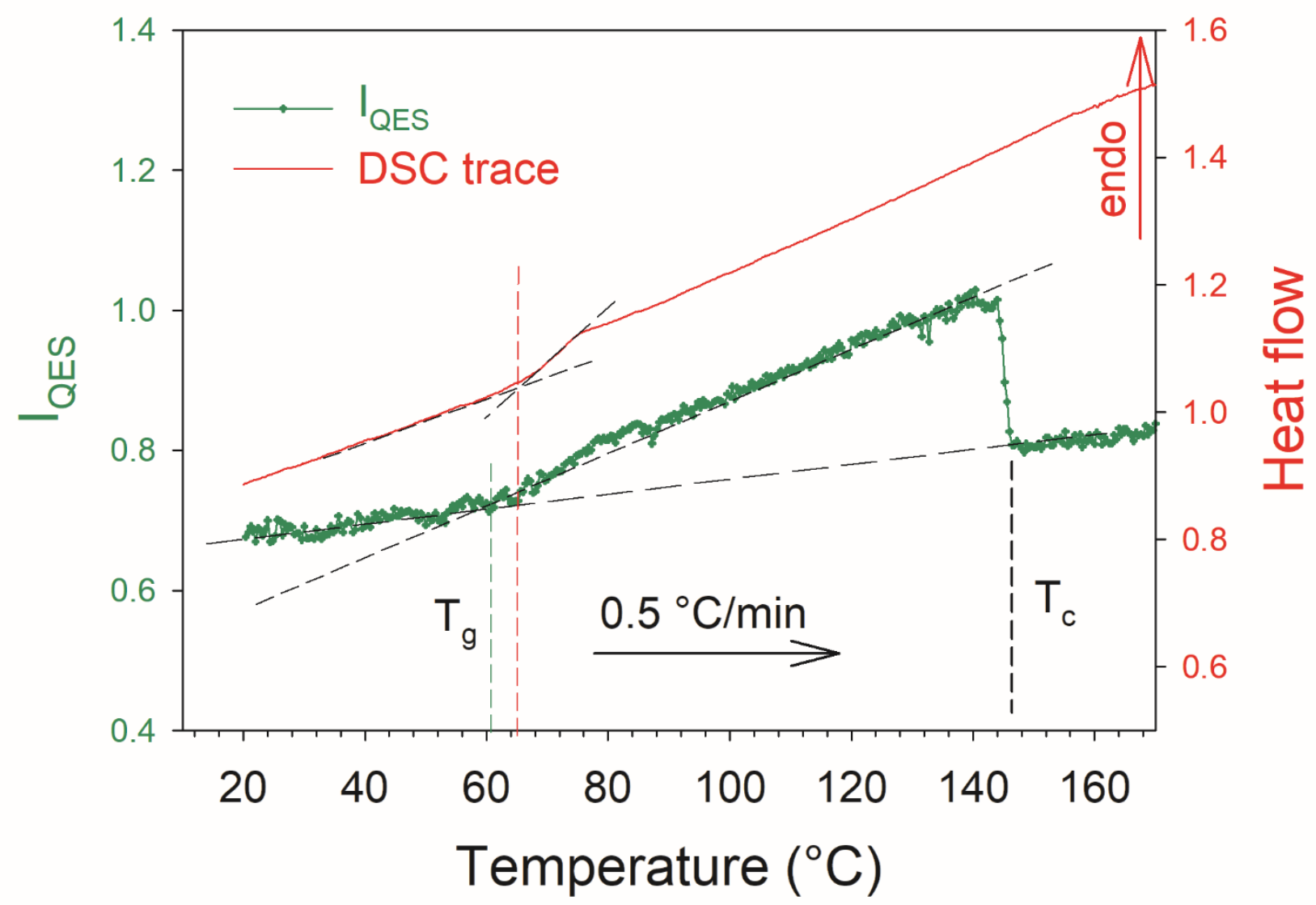

Figure 2: comparison of the temperature dependence of the quasielatic intensity and the DSC traces obtained by heating the glassy state obtained by quenching the liquid state down to room temperature 

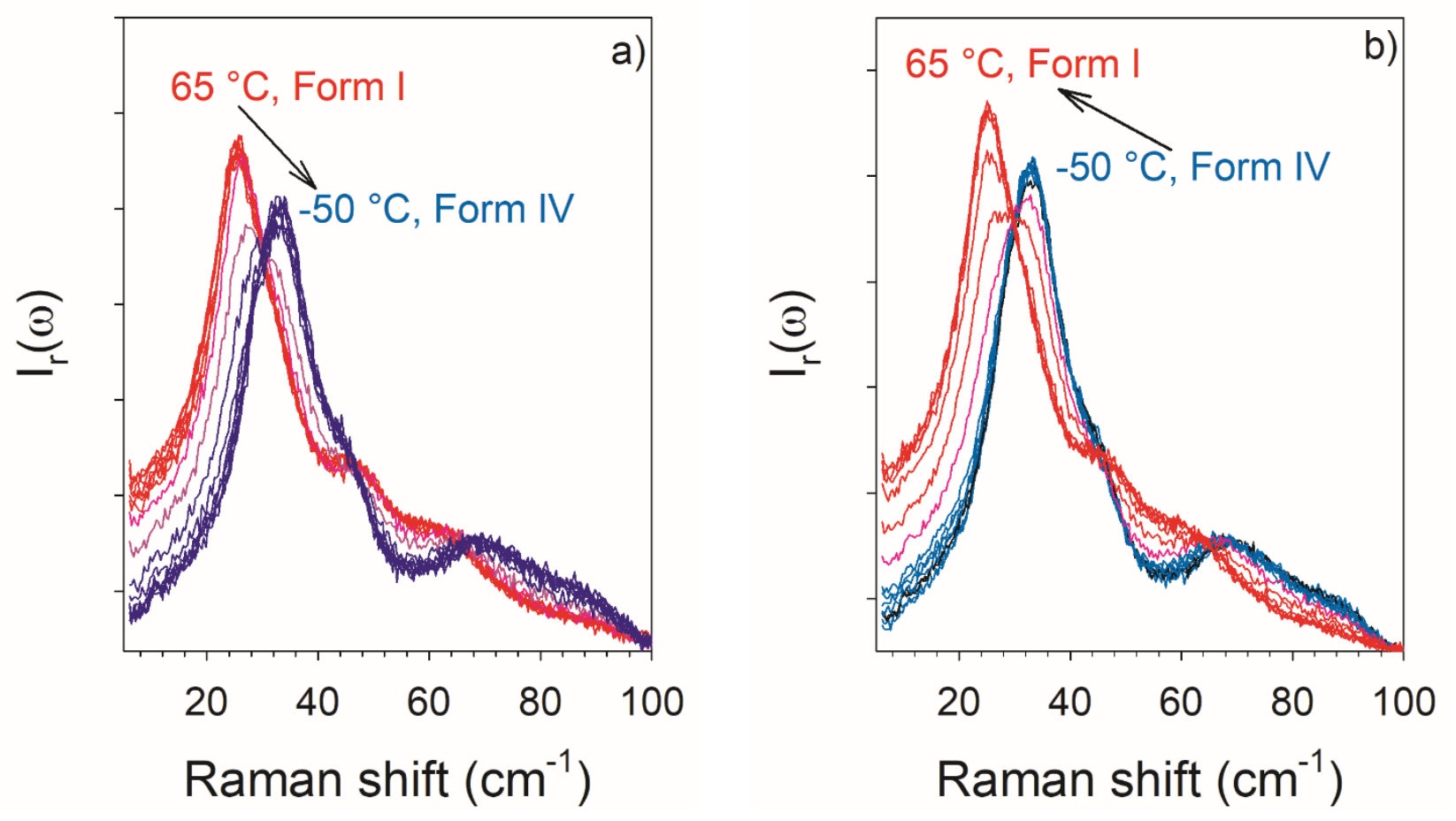

Figure 3: temperature dependence of $\operatorname{Ir}(\omega)$ spectra, calculated from spectra collected during

a) cooling ramp of Form I at $\dot{T}=-0.5^{\circ} \mathrm{C} / \mathrm{min}$

b) heating ramp of Form III at $\dot{T}=0.5^{\circ} \mathrm{C} / \mathrm{min}$

Form I was obtained from devitrification of the quenched liquid at $140{ }^{\circ} \mathrm{C}$ 


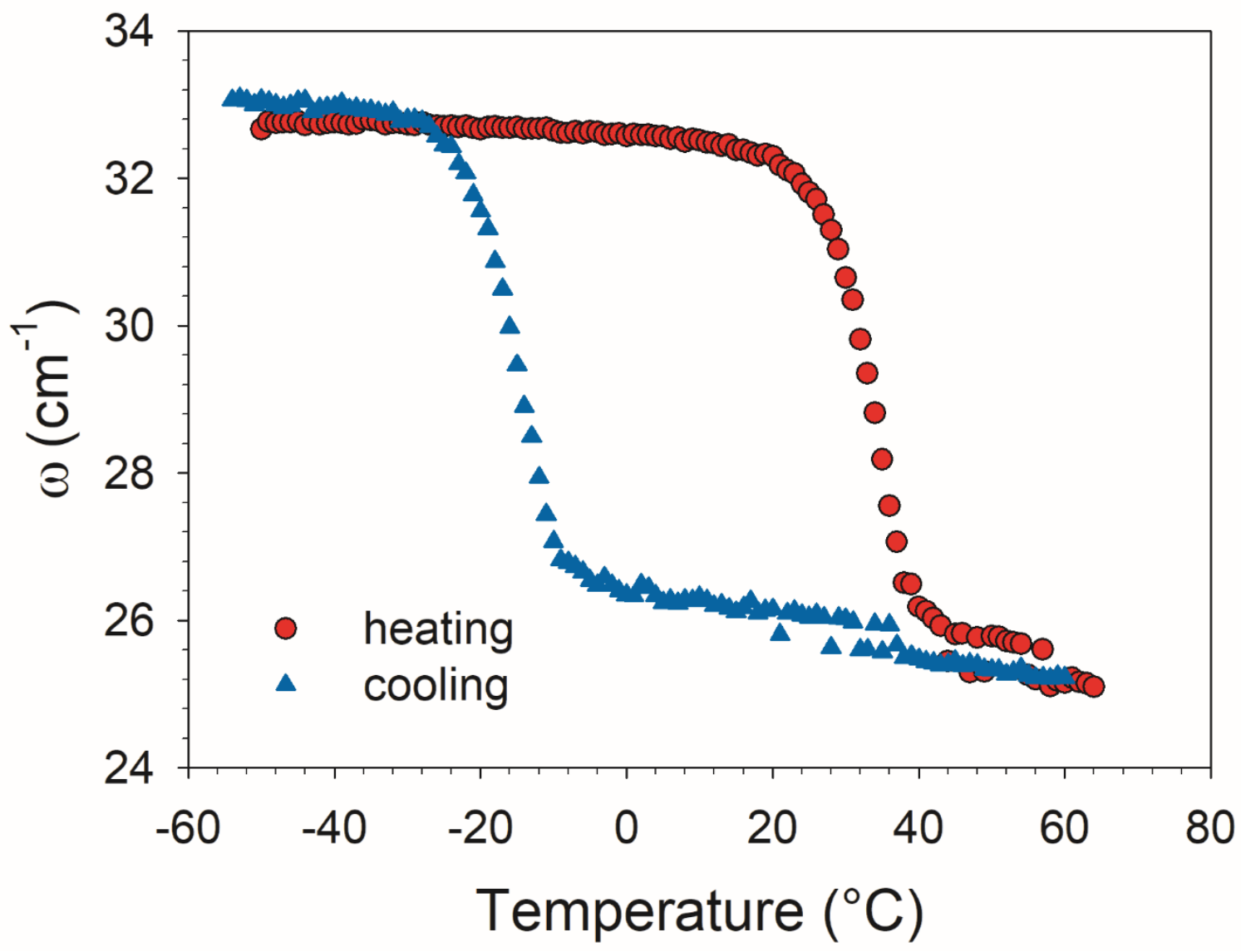

Figure 4: temperature dependence of the position of the lowest wavenumber band upon cooling and heating at $\dot{T}=0.5^{\circ} \mathrm{C} / \mathrm{min}$ 

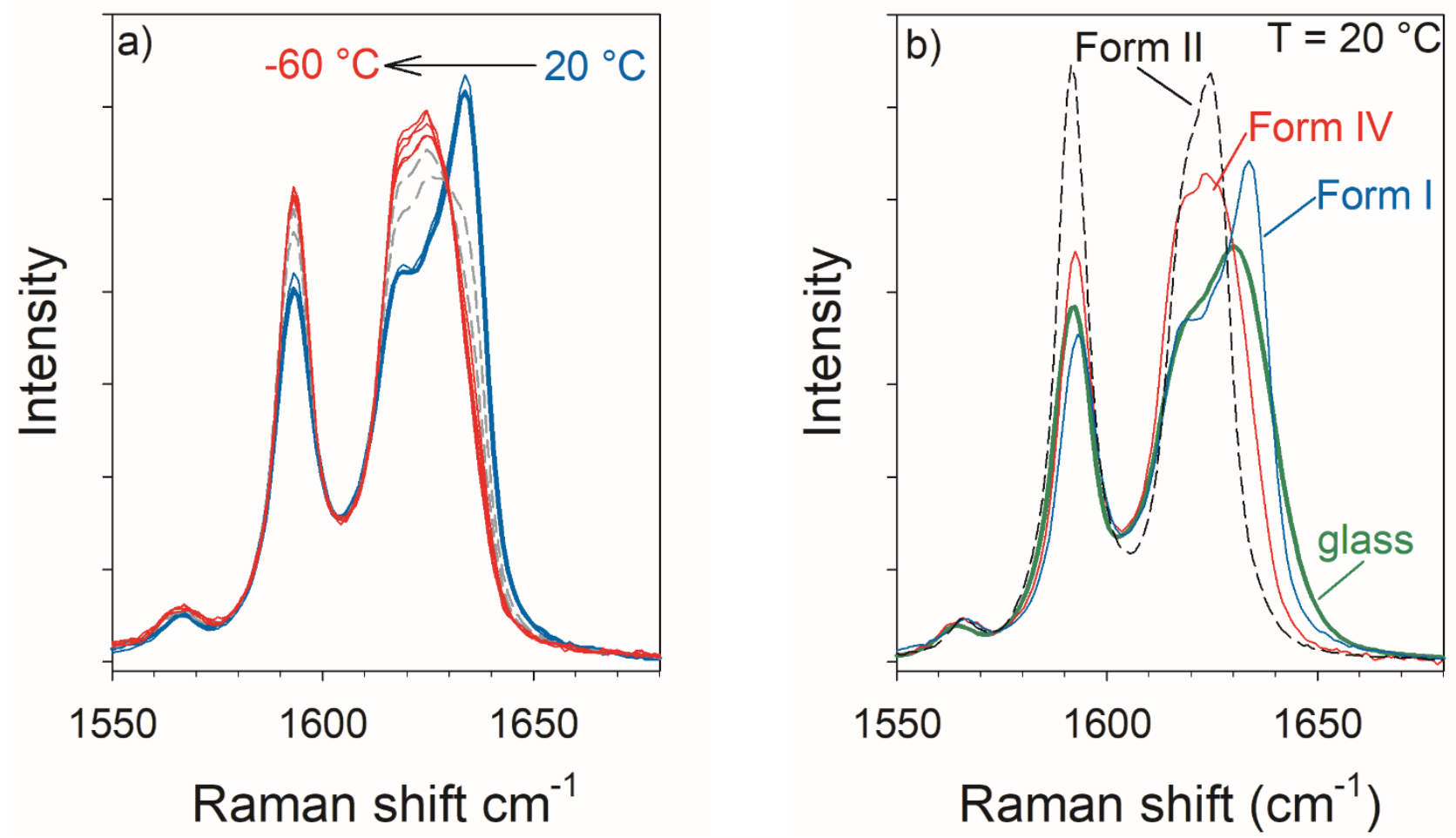

Figure 5: Raman spectra in the fingerprint region collected

a) during Form I to Form IV transformation;

b) in the different states of sulindac at $20^{\circ} \mathrm{C}$ 

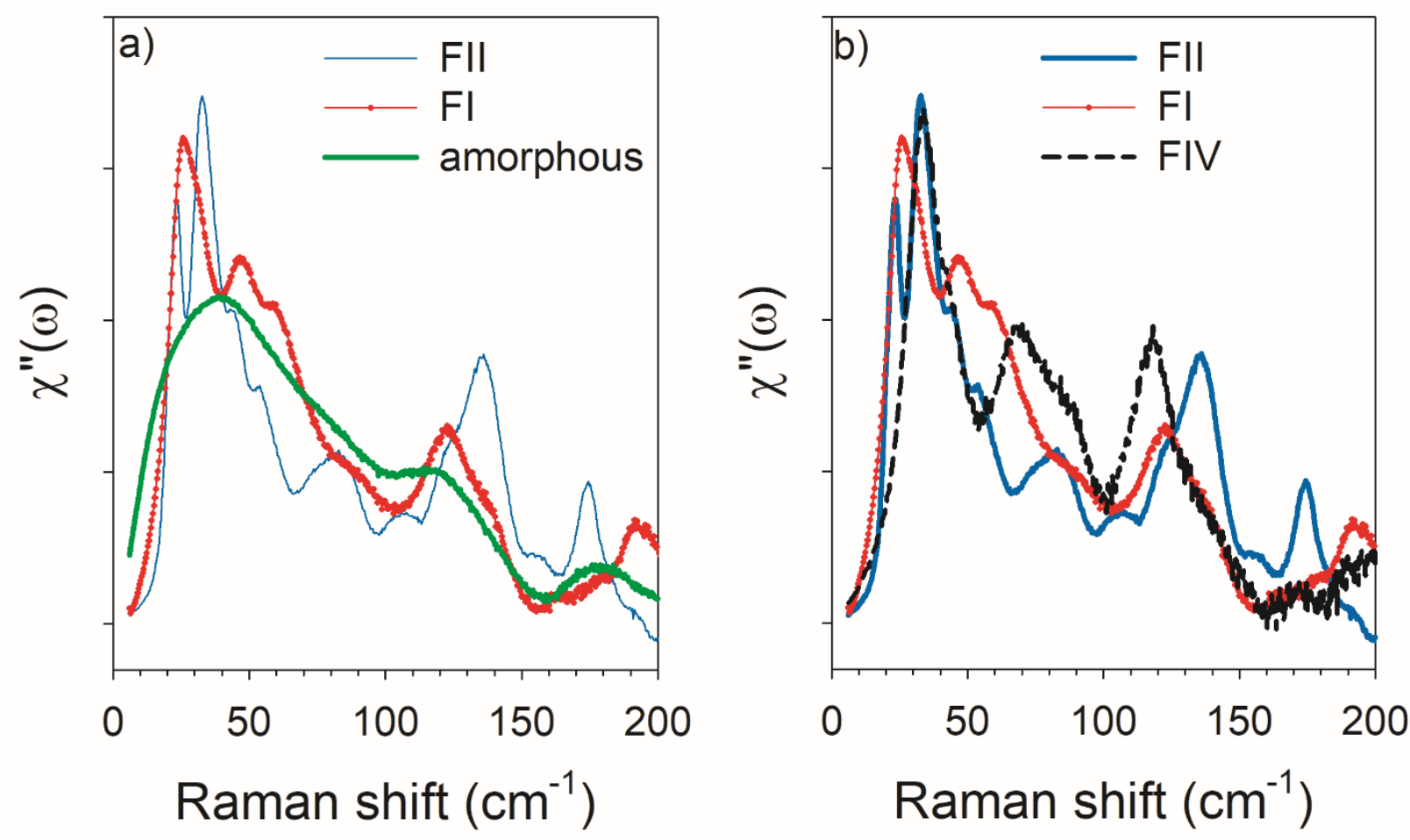

Figure 6: Representation of Raman susceptibilities of the amorphous and crystalline states of sulindac

a) comparison of Forms I and II in relation with the amorphous state

b) comparison of new Form IV with Forms I and II 


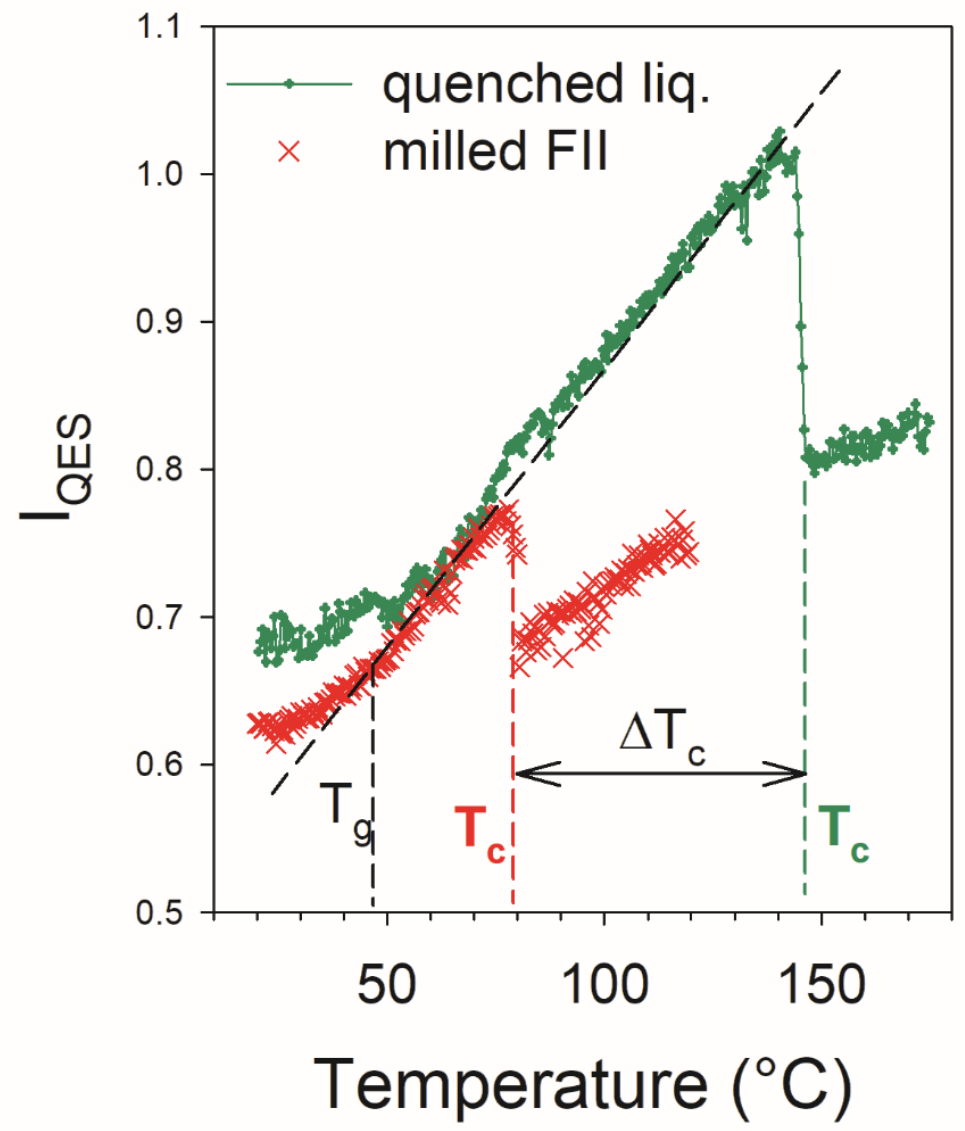

Figure 7: comparison of $\mathrm{I}_{\mathrm{QES}}(\mathrm{T})$ determined from low-wavenumber spectra collected upon heating at $0.5^{\circ} \mathrm{C} / \mathrm{min}$ quenched liquid and amorphous powder obtained by milling Form II for 600 minutes 


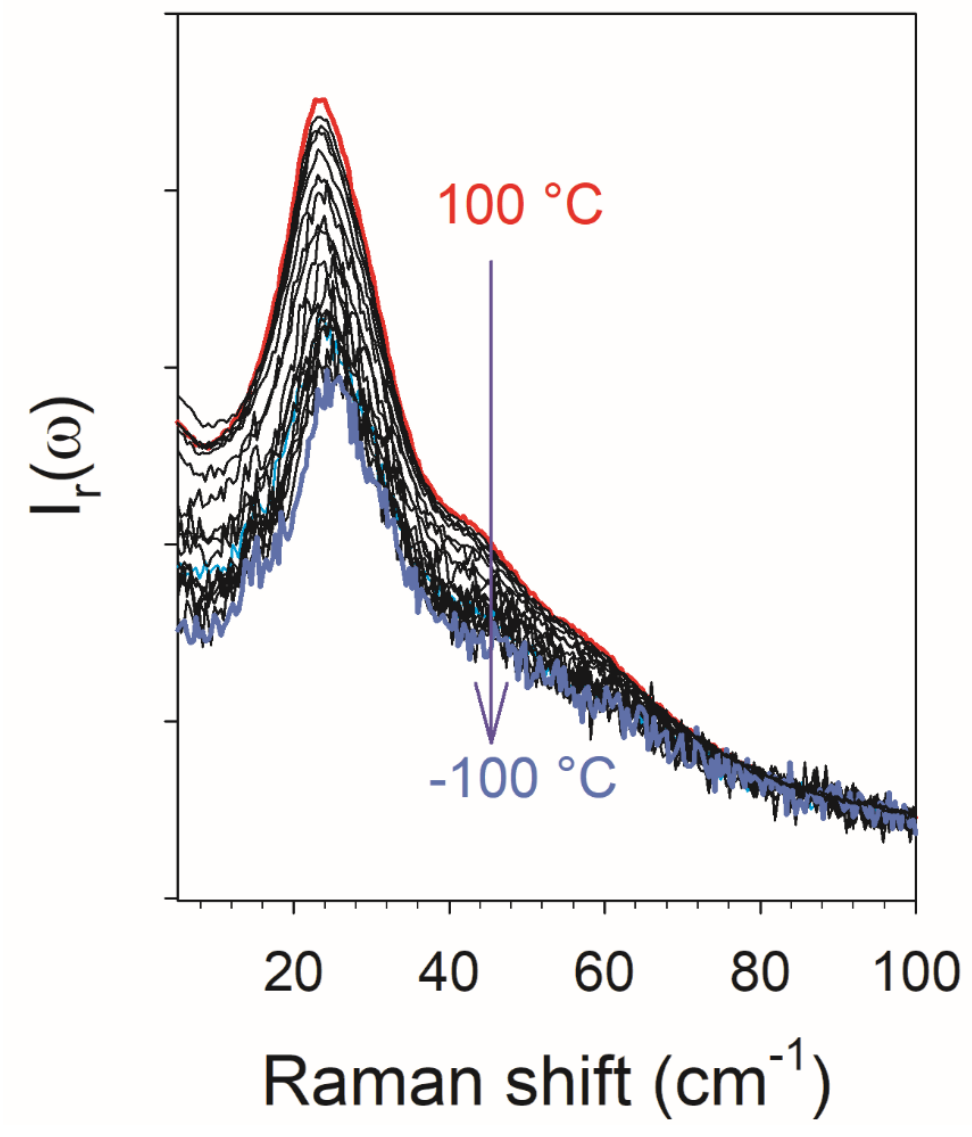

Figure 8: temperature dependence of $\mathrm{I}_{\mathrm{r}}(\omega)$ spectra calculated from spectra collected during a cooling ramp of Form I obtained by devitrification of the amorphous milled powder.

Crystallization of Form I was detected at $70{ }^{\circ} \mathrm{C}$, and was heated up to $100{ }^{\circ} \mathrm{C}$ before cooling 


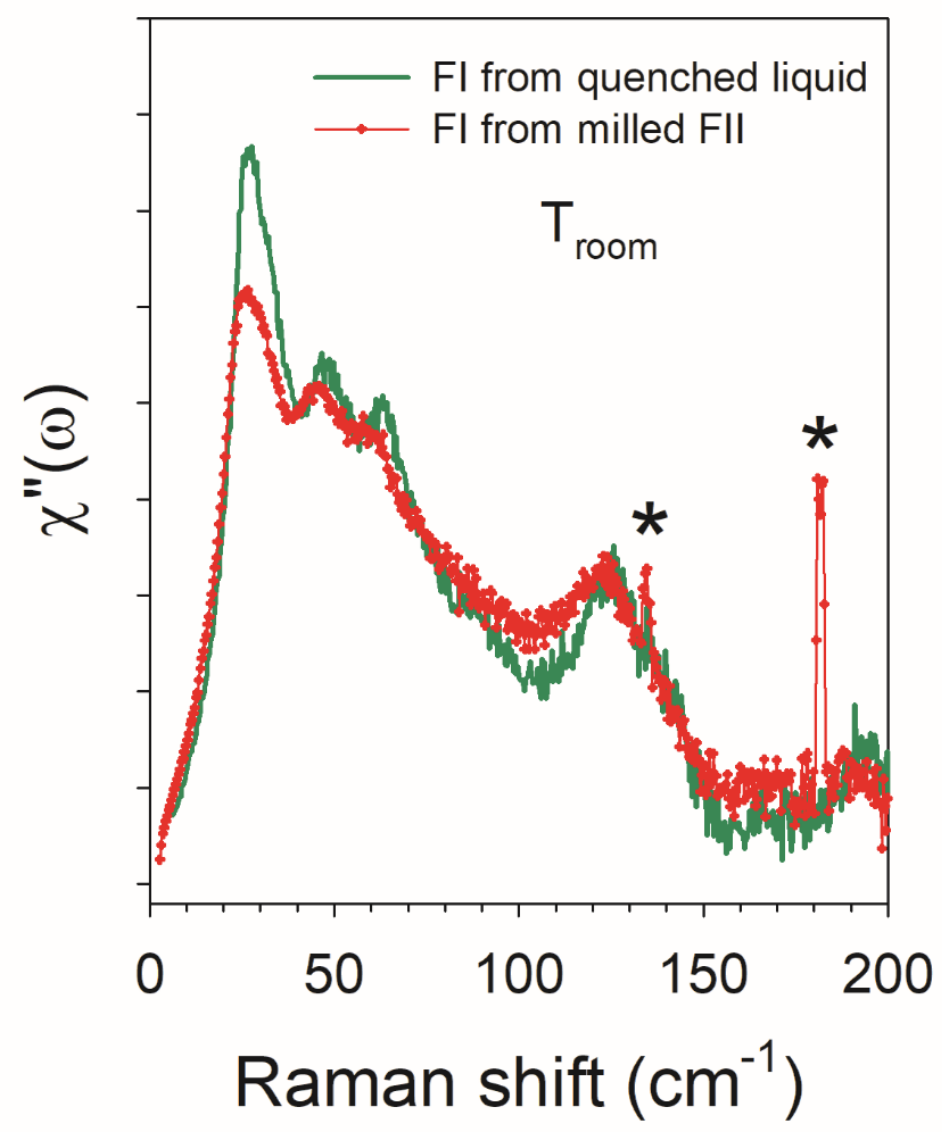

Figure 9: comparison of Raman susceptibility spectra of Form I prepared by devitrification of quenched liquid and by devitrification of amorphous powder obtained by milling Form II. Stars indicate laser lines. 